\title{
Targeted CRISPR/Cas9-Based Knock-Out of the Rice Orthologs TILLER ANGLE CONTROL 1 (TAC1) in Poplar Induces Erect Leaf Habit and Shoot Growth
}

\author{
Matthias Fladung
}

check for updates

Citation: Fladung, M. Targeted CRISPR/Cas9-Based Knock-Out of the Rice Orthologs TILLER ANGLE CONTROL 1 (TAC1) in Poplar Induces Erect Leaf Habit and Shoot Growth. Forests 2021, 12, 1615. https://doi.org/10.3390/f12121615

Academic Editor: Dirk Landgraf

Received: 27 October 2021

Accepted: 19 November 2021

Published: 23 November 2021

Publisher's Note: MDPI stays neutral with regard to jurisdictional claims in published maps and institutional affiliations.

Copyright: (C) 2021 by the author. Licensee MDPI, Basel, Switzerland. This article is an open access article distributed under the terms and conditions of the Creative Commons Attribution (CC BY) license (https:/ / creativecommons.org/licenses/by/ $4.0 /)$.
Thünen Institute of Forest Genetics, Sieker Landstrasse 2, 22927 Grosshansdorf, Germany; matthias.fladung@thuenen.de; Tel.: +49-41-0269-6107

\begin{abstract}
Pyramidal-, erect- or upright-growing plant forms are characterized by narrow branch angles of shoots and leaves. The putative advantage of upright-leaf and shoot habit could be a more efficient penetration of light into lower canopy layers. Pyramidal genotypes have already been reported for various tree genotypes including peach. The paralogous rice ortholog TILLER ANGLE CONTROL 1 (TAC1) has been proposed to be the responsible gene for upright growth. However, it has not really been demonstrated for any of the pyramidal tree genotypes that a knock-out mutation of the TAC1 gene is causing pyramidal plant growth. By in silico analyses, we have identified a putative rice TAC1 ortholog (Potri.014G102600, "TAC-14") and its paralog (Potri.002G175300, "TAC-2") in the genome of $P$. trichocarpa. Two putative PCTAC1 orthologs in the $P . \times$ canescens clone INRA 717-1B4 were successfully knocked-out by applying a transgenic CRISPR/Cas9-approach. The mutants were molecularly analyzed and phenotyped over a period of three years in a glasshouse. Our results indicate that the homozygous knock-out of "TAC-14" is sufficient to induce pyramidal plant growth in $P . \times$ canescens. If up to twice as many pyramidal individuals were planted on short rotation coppices (SRCs), this could lead to higher wood yield, without any breeding, simply by increasing the number of trees on a default field size.
\end{abstract}

Keywords: CRISPR/Cas9; genome editing; Populus; INRA 717-1B4; pyramidal plant habitus; leaf petiole angle; branch angle

\section{Introduction}

Plants possess variable plant architectures and phenotypes which facilitate their optimal adaptation to specific environments, even if these do not always offer the best growing conditions. Plant architecture is species-specific and, therefore, has been used for decades as the basis for systematics and the taxonomic classification of plants [1]. However, variability of the plant phenotype and the degree to which the branching pattern is dependent on the growing habitat is well known and has already fascinated geneticists, developmental biologists, and plant breeders for more than 100 years. Plant architecture, in general, is characterized by the internodial and nodial organization of the main shoot, and location, orientation, and number of side-shoots, influenced by genetic components [1-6], and modulated by developmental and environmental factors like gravity, light, water, and touch $[7,8]$.

An uncountable number of reports have been published in the past describing the spontaneous or induced alteration of the genetic constitution of an individuum, i.e., random or targeted mutagenesis mainly applied for the improvement of human food, e.g., barley [9] but also ornamental plants [10-14]. Here, artificial induction of mutations by X-ray irradiation or chemical treatments has been a key element of mutation breeding. Many of the mutant individuals obtained but also natural mutants showed alterations in plant habitus and architecture, e.g., nana, compacta, fastigiata, pendula, and pyramidalis (e.g. [14-17]). 
Only very few mutations are dominant and become visible in a heterozygous allelic status. However, most mutations are of recessive nature and require allele homozygosity for phenotypic expression. Thus, a self-pollination cycle is needed for the generation of mutants. However, the timespan for vegetative growth until the generative phase is reached is, in particular in forest trees, usually very long, i.e., ranges from a few years to several decades. A solution to circumvent this problem is the application of modern biotechnological approaches, like genetic engineering or genome editing technology by transferring or modifying key genes. Both technologies offer a straight-forward way to alter plant architecture in a manageable timeframe in trees [18].

For example, the creation of dwarf and semi-dwarf mutants has been described following the genetic transfer of genes modifying hormone biosynthesis, e.g., gibberellic acid (GA), or conferring GA insensibility [19,20], or by hyperactivation of the GA2-OXIDASE (GA2ox) gene, the major GA catabolic enzyme in plants, in an activation tagging approach [21]. Dwarf poplar mutants have also been reported after transfer of the meristem identity gene LEAFY (LFY) [22,23], the TaLEA (Tamarix androssowii LATE EMBRYOGENESIS ABUNDANT GENE) [24], of two flowering time genes (SUPPRESSOR OF CONSTANS [SOC1] and FRUITFUL [FUL] [25], the ROLC gene from Agrobacterium rhizogenes [26-29], or by adopting an Ac/Ds-transposon activation tagging-based mutation system in poplar [30]. On the other hand, poplar with increased plant size have also been reported after e.g., transfer and overexpression of the GA20-OXIDASE gene [31-33] or knock-down of PHENYLCOUMARAN BENZYLIC ETHER REDUCTASE1 (PCBER1), a gene of the neolignan biosynthesis pathway [34].

Pyramidal or erect plant growth has become of interest for commercial applications because of its possible advantage in plant yield, as reported for rice [35] and spring wheat [36]. Characteristic for the upright-growing branches is the narrow branch angle of shoots and leaves. An advantage of pyramidal growth could be that the erect-leaf habit allows more efficient penetration of light into lower canopy layers, and thus increases photosynthetic potential of the whole plant [35,37]; for review: [38]. Other papers, however, stated that factors like nitrogen and water availability more likely influence plant and grain yield [39]. But independently of possible physiological potential associated with erect leaf or shoot growth, plants with reduced need for individual space on a field would simply allow a higher number of plants to be planted on a default field size, as e.g., reported for Prunus persica (peach) [40] and Malus domestica (apple) [41].

In peach, mutant varieties with pronounced vertical growth of branches or upright stems have been investigated in detail $[42,43]$. This trait, originally named "broomy" (br) and later re-named "pillar", has been described as intermediate because heterozygous offspring revealed branch angles in between the two extremes [43]. It has been shown that the broomy or pillar phenotype is caused by a loss-of-function mutation in the ortholog of the rice TILLER ANGLE CONTROL 1 (TAC1) gene $[43,44]$. On the other hand, overexpression of the PpTAC1 gene in plum caused wider branch angles resulting in more horizontal oriented branches [44]. TAC1 belongs to the IGT gene family which is characterised by a $\mathrm{G} \varphi \mathrm{L}(\mathrm{A} / \mathrm{T}) \mathrm{IGT}$ motif [43]. This family contains other well-known members like LAZY1 and DEEPER ROOTING 1 (DRO1).

Other prominent examples showing pyramidal plant growth are e.g., Cupressus sempervirens 'Pyramidalis' (Italian Cypress [45]), Taxus baccata 'Pyramidalis' (pillar yew [46]), Populus tremula 'Erecta' (Swedish columnar aspen [47]), P. alba var. pyramidalis (P. bolleana [48]), and P. nigra cv. 'Italica' (Lombardy poplar [49]). Also, the commercial AC Sundancer ${ }^{\mathrm{TM}}$ hybrid poplar cultivar demonstrates vertical growth of branches [50]. For Lombardy poplar, ref. [43] has proposed that the pyramidal phenotype is possibly caused by a mutation in a poplar ortholog of TAC1. Interestingly, this pyramidal mutant poplar was detected in Central Asia in the 19th century [49], and since then it was propagated vegetatively on a very large scale and distributed worldwide as a single genotype [51,52]. However, for both, Lombardy poplar and for Swedish columnar aspen, or for any other 
pyramidal tree genotype, it has not really been demonstrated that a knock-out mutation of the poplar TAC1-orthologous gene is the cause of pyramidal plant growth.

In in silico studies, we have identified a putative rice TAC1 ortholog in the P. trichocarpa genome and because of the quite recent complete genome duplication in poplar [53], its most homologous paralog. Based on these two PtTAC1 sequences, we also determined the two putative TAC1 homologous genes PtrTAC1 and PCTAC1 in the genomes of P. tremula and $P . \times$ canescens. To prove that one or the other, or both, TAC1 ortholog(s) are responsible for pyramidal plant growth in poplar, we applied the CLUSTERED REGULARLY INTERSPACED SHORT PALINDROMIC REPEAT (CRISPR)/CRISPR-associated protein 9 (Cas9) editing system [54] to knock-out the two putative PcTAC1 orthologs in the INRA 717-1B4 clone. We could show that the homozygous knock-out of only Potri.014G102600 ("TAC-14") is sufficient to induce pyramidal plant growth in $P$. $\times$ canescens.

\section{Materials and Methods}

\subsection{Plant Material}

The hybrid poplar clone INRA 717-1B4 or "P1" $(P . \times$ canescens $=$ P. tremula $\times$ P. alba [55], grown in vitro on WPM medium without hormones at $23^{\circ} \mathrm{C}$ and with $24 \mathrm{~h}$ light was used for Agrobacterium-mediated leaf-disc transformation (see below). Putative transgenic plants were regenerated after four to eight weeks culture on kanamycin-containing regeneration medium as described in [56]. Regenerated but not-transgenic plants (without any PcTAC1 editing), as well as P1 from tissue culture, served as controls.

\subsection{Identification of Putative PpeTAC1 Homologs in Poplar}

Following blast of the coding sequence of the Prunus persica gene PpeTAC1 (EMBL acc.no. KF218366; [43], in the full genome sequence of P. trichocarpa v3.1 (https:/ / phytozome-next. jgi.doe.gov/info/Ptrichocarpa_v3_1, accessed on 22 November 2021), two significant hits were obtained: Potri.014G102600 (score: 183.4, E-value: $8.7 \times 10^{-44}$ ) and Potri.002G175300 (score: 172.6; E-value: $1.6 \times 10^{-40}$ ) (Supplementary Materials Table S1). The third hit was on chromosome 18 but with just a score of 46.4 and E-value of $1.6 \times 10^{-2}$. Therefore, we considered the two best hits as paralogous genes for the CRISPR/Cas-induced mutation approach.

The transcript sequences of Potri.014G102600 and Potri.002G175300 were blasted against the draft genome sequence of $P . \times$ canescens (clone INRA 717-1B4; P1;https:// urgi.versailles.inra.fr/Species/Forest-trees/Populus/Clone-INRA-717-1B4, accessed on 22 November 2021) and P. tremula v1.1 PopGenIE (http:/ / popgenie.org/blast, accessed on 22 November 2021), respectively, to find any putative homologous sequences for the used clone P1. The blast-results indicate that the Potri.014G102600 and Potri.002G175300 homologous sequences in $P . \times$ canescens are scaffold_72118, scaffold_37509, scaffold_1688, scaffold_170684 and scaffold_333464, scaffold_74020, scaffold_47155, respectively, and in P. tremula are Potra003475 and Potra001079, respectively (Supplementary Materials Table S1).

Obtained sequences were aligned to find identical positions in the first three exons of all TAC1 homologs in P. trichocarpa, $P . \times$ canescens and $P$. tremula where target sequences in the gRNAs for simultaneous knock-out of both genes can be designed.

\subsection{Guide RNA ( $g R N A$ ) Design and Agrobacterium-Mediated Poplar Transformation}

We employed the CRISPR/Cas9 system to generate TAC1 mutations. Two guide RNAs (gRNAs) were designed manually. Secondary structures of the applied gRNAs were subsequently analysed with the RNAfold WebServer [57], accessible under http: //rna.tbi.univie.ac.at/cgi-bin/RNAWebSuite/RNAfold.cgi (accessed on 30 October 2020), as described in [54]. This software relies on the RNA folding model of [58] and the minimum free energy (MFE) computing algorithm of [59].

Putative PpeTAC1 (EMBL acc.no. KF218366; [43]) homologous genes in P. trichocarpa, $P$. $\times$ canescens and $P$. tremula were identified. For the CRISPR/Cas9-approach, two target sequences of the gRNAs were selected in exon 3 of the two paralogs Potri.014G102600 and 
Potri.002G175300 (for each paralog 2R and 4F) (P. trichocarpa v3.1 (https:/ / phytozome-next. jgi.doe.gov/info/Ptrichocarpa_v3_1, accessed on 22 November 2021). The reason to design two gRNAs was simply to double the chance of a successful editing. Both target regions are located in exon 3 of the two genes, only a few hundred base pairs apart.

Care was taken that both target sequences were mapped to identical positions in the two TAC1 paralogs to ensure simultaneous knock-out of both genes. In addition, target sequences were checked for homology to the genomic sequence of used P1 in AspenDB (http://aspendb.uga.edu/index.php/databases/spta-717-genome, accessed on 22 November 2021) and (https://urgi.versailles.inra.fr/Species/Forest-trees/Populus/ Clone-INRA-717-1B4, accessed on 22 November 2021 [60], and P. tremula (PopGenIE; http:/ / popgenie.org/blast, accessed on 22 November 2021). The following two target sequences were chosen: (i) TAC1_4F_gRNA (target: CAC TGG CAG AAC TGT TCT TGG): in exon 3 of both Potri.002G175300 (13,448, 819 to 13,448,839) and Potri.014G102600 $(8,029,047$ to $8,029,067)$, (ii) TAC1_2R_gRNA (target: CCA TCA AGC ACA TCC ACC AGG): in exon 3 of both Potri.002G175300 $(13,449,184$ to 13,449,204) and Potri.014G102600 $(8,029,382$ to $8,029,402)$. The assembled multiplexing vector C672p9ioR-35s-CasWT-Nuc (14,257 bp; Supplementary Materials Figure S1) was transferred into Agrobacterium tumefaciens strain GV3101 (DNA Cloning Service, Hamburg, Germany) which was used for poplar leaf-disc transformation as described above. Details of the vector, enzymes, and antibiotics for Agrobacterium-growth are mentioned in the website of the company (https: / / dna-cloning.com/vectors/, accessed on 22 November 2021).

Plant transformation was carried out by applying the Agrobacterium-mediated leaf-disc co-cultivation method as described in $[28,29,61]$. Leaf-discs of in vitro grown P1-plants were harvested and soaked with Agrobacterium tumefaciens strain GV3101::pMP90RK (chromosomal background C58) carrying the CRISPR/Cas9 system (35S-driven Cas9 nuclease and gRNAs: C672p9ioR-35sCasWT-Nuc, Supplementary Materials Figure S1). The plasmid (provided by DNA Cloning Service, Hamburg, Germany) is also carrying the NEOMYCIN PHOSPHOTRANSFERASE II (NPTII) gene leading to kanamycin (kan) resistance. For transgenic plant selection, the regeneration media contained kanamycin $(50 \mathrm{mg} / \mathrm{L})$, and cefotaxime (500 $\mathrm{mg} / \mathrm{L}$ ) for bacterium removal [56].

\subsection{Molecular Analyses of Transgenic Plants and Sequencing of CRISPR/Cas9-Induced Mutations}

Genomic DNA was extracted from leaves of the 19 different putative Cas9, TAC1_4F_ gRNA, and TAC1_2R_gRNA transgenic lines of the line N499 and the not-transgenic P1-control grown in vitro for PCR analyses according to [28,32]. PCR was performed as described in detail in [29] but with annealing temperatures of $58{ }^{\circ} \mathrm{C}$ for amplification of a partial Cas9-fragment and $60^{\circ} \mathrm{C}$ for NPTII. The sequences of the primer pairs used in PCR reactions to amplify fragments of the Cas9 and the NPTII genes are listed in Supplementary Materials Table S2.

CRISPR/Cas9-related mutations in the $P . \times$ canescens paralogs Potri.014G102600 and Potri.002G175300 were determined by Sanger sequencing of the two genomic target sites (for each paralog $2 \mathrm{R}$ and $4 \mathrm{~F}$ ). Primers used are summarized in Supplementary Materials Table S1. The mutations were homozygous and heterozygous single and double nucleotide insertions or deletions in one or both paralogous genes.

\subsection{Morphological Investigations of Glasshouse Grown Plants}

To experimentally test whether CRISPR/Cas9-mediated homozygous or heterozygous mutations in the $P . \times$ canescens paralogs Potri.014G102600 and Potri.002G175300 reveal a modified plant growth or habitus, plants from transgenic lines and controls, including two not-transgenic but in vitro regenerated lines (without any TAC1-mutation) and the wildtype clone P1, were multiplied in vitro by shoot cuttings.

Following in vitro culture, rooted shoots of about 5 to $10 \mathrm{~cm}$ in height were potted into soil in April 2019, transferred to growth chambers, fully covered with plastic lids, and cultivated in boxes on a $25^{\circ} \mathrm{C} / 16^{\circ} \mathrm{C}$ and $16 / 8 \mathrm{~h}$ day/night cycle. For acclimatization to 
ambient air conditions (relative humidity of air: 70\%), lids were opened daily for increasing time periods over one week. Following acclimatization, plants were watered daily and cultivated for 2 to 4 weeks in the growth chamber. At an age of about 4 to 6 weeks, plants were transferred into the glasshouse (min-mean-max air temperature, April to October: 15-19-24 ${ }^{\circ} \mathrm{C}$, November to March: $8-17-21^{\circ} \mathrm{C}$ ). During the first and second growing season, the plants were cultivated in pots with sizes of 13 and $26 \mathrm{~cm}$, respectively, watered daily, and supplemented with mineral fertilizer twice during the growing season.

Total height and diameter of the main shoot $(1 \mathrm{~cm}$ above soil) were measured from five plants of each of the ten independent transgenic lines N499-1-2, -2-1, -3-1, -4-1, -8-3, -10-2, $-11-1,13-2,-14-1$, -and 17-1, the control P1, and the regenerated but not-transgenic lines N499-6-1 and -9-1, cultivated in glasshouse after terminal bud formation (January 2020 and January 2021). Three to ten leaf petioles (July 2019) and side-shoots (August 2020 and April 2021) were photographed from five plants of each of the ten independent N499transgenic lines mentioned before, the regenerated but not-transgenic lines and the control P1. Later, angles of leaf petioles and side-shoots were determined on a computer screen.

\subsection{Data Analysis and Statistics}

Data are presented in figures as mean + SD. Differences of the last measurements were tested for significance by ANOVA at $p<0.05$ (Fisher's F-test). Values in the figures labelled with an asterisk differ significantly at $p<0.05$ (Fisher's F-test) from the control.

\section{Results}

\subsection{Regeneration and Molecular Analysis of Putative TAC1 Mutants}

In total, 19 independent putatively transgenic lines were obtained after four to eight weeks on kanamycin-containing regeneration medium in the transformation experiment N499. Regenerated lines were termed as N499-X. All lines were investigated via PCR for presence of NPTII and Cas9 genes (Table 1). The results indicate that out of the 19 independent lines, ramets of eleven lines revealed both the NPTII and the Cas9 gene (Table 1).

Table 1. PCR analyses amplifying parts of the NPTII and Cas 9 genes of putative transgenic lines carrying the CRISPR/Cas9 system to modify the genomic sequences of the P. trichocarpa homologs Potri.014G102600 and Potri.002G175300. + = band appeared in PCR-experiments; - = no band.

\begin{tabular}{cccc}
\hline Putative Transgenic Line & Ramet & NPTII & Cas9 \\
\hline N499-1 & -2 & + & + \\
\hline N499-2 & -1 & + & + \\
\hline N499-3 & -1 & + & + \\
\hline N499-4 & -1 & - & - \\
\hline N499-5 & -1 & - & - \\
\hline N499-6 & -1 & - & + \\
\hline N499-7 & -1 & + & + \\
\hline N499-8 & -3 & - & + \\
\hline N499-9 & -1 & + & - \\
\hline N499-10 & -2 & + & + \\
\hline N499-11 & -1 & - & + \\
\hline$N 499-12$ & -1 & + & - \\
\hline$N 499-13$ & -2 & + & + \\
\hline$N 499-14$ & -2 & -1 & + \\
\hline$N 499-15$ & & + & + \\
\hline & -15 & + & + \\
\hline
\end{tabular}


Table 1. Cont.

\begin{tabular}{cccc}
\hline Putative Transgenic Line & Ramet & NPTII & Cas9 \\
\hline N499-16 & -1 & - & - \\
\hline N499-17 & -1 & + & + \\
\hline N499-18 & -1 & - & - \\
\hline N499-19 & -1 & + & + \\
\hline
\end{tabular}

The two target sites 2R and 4F in the paralog Potri.014G102600 were Sanger-sequenced in all obtained lines (Tables 2 and 3). The results indicate that all lines tested positive for NPTII and Cas9, PCR also reveal CRISPR/Cas9-caused mutations in the target 2R (Tables 2 and 3). Here, a preferential homozygous insertion of one single nucleotide was found, mostly T, but also A or G, or in lines N499-4-1, -8-3, -11-1 a different nucleotide in the two allelic strands, namely K (Table 3). In line N499-1-2, a deletion of two nucleotides was found. An undefinable heterozygous insertion or deletion was detected in line N499-13-2. On the other hand, not a single mutation in any line could be identified in the target $4 \mathrm{~F}$.

Table 2. Sanger sequencing of the two target regions of the two P. trichocarpa putative PpeTAC1 paralogs Potri.014G102600 and Potri.002G175300 in P. $\times$ canescens (for each paralog targets $2 \mathrm{R}$ and $4 \mathrm{~F}$ ) to unravel putative CRISPR/Cas9-caused mutations. - = no modification (similar to P1), similar to wildtype control clone P1, del = deletion, ins = insertion, hom = homozygous, het $=$ heterozygous, $?=$ not definable, n.d. = not determined. Grey shaded lines in the first column were transferred to the glasshouse for morphological inspections. Bold in Potri: lines with homozygous mutations in both genes. Underlined in Potri: lines with heterozygous mutations in both genes. Grey shaded, bold, and underlined in Potri: lines with homozygous mutation in Potri.014G102600 but with heterozygous mutation in Potri.002G175300.

\begin{tabular}{|c|c|c|c|c|}
\hline \multirow[t]{2}{*}{ P1/Putative Transgenic Line } & \multicolumn{2}{|c|}{ Potri.014G102600 (“TAC-14") } & \multicolumn{2}{|c|}{ Potri.002G175300 ("TAC-2") } \\
\hline & $2 R$ & $4 \mathrm{~F}$ & $2 R$ & $4 \mathrm{~F}$ \\
\hline P1 (Wildtype) & - & - & - & - \\
\hline N499-1-2 & GT del hom & - & G ins hom & - \\
\hline N499-2-1 & T ins hom & - & $R$ ins hom & - \\
\hline N499-3-1 & $T$ ins hom & - & $R$ ins hom & - \\
\hline N499-4-1 & K ins hom & - & ? ins/del het & - \\
\hline N499-5-1 & - & - & n.d. & - \\
\hline N499-6-1 & - & - & - & - \\
\hline N499-7-1 & - & - & n.d. & - \\
\hline N499-8-3 & K ins hom & - & $R$ ins hom & - \\
\hline N499-9-1 & - & - & - & - \\
\hline N499-10-2 & T ins hom & - & A ins hom & - \\
\hline N499-11-1 & K ins hom & - & ? ins/del het & - \\
\hline N499-12-1 & - & - & n.d. & - \\
\hline N499-13-2 & ? ins/del het & - & ? ins/del het & - \\
\hline N499-14-2 & T ins hom & - & ? ins/del het & - \\
\hline N499-15-1 & - & - & n.d. & - \\
\hline N499-16-1 & - & - & - & - \\
\hline N499-17-1 & T ins hom & - & ? ins/del het & - \\
\hline N499-18-1 & - & - & - & - \\
\hline N499-19-1 & $\mathrm{T}$ ins hom & - & K ins hom & - \\
\hline
\end{tabular}


Table 3. Types of mutations identified in the target region $2 \mathrm{R}$ of the two $P$. trichocarpa putative PpeTAC1 paralogs Potri.014G102600 and Potri.002G175300 in P. $\times$ canescens following CRISPR/Cas9-editing. Heterozygous mutations were complex and were not resolvable in detail. Underlined: target sequence 2R (CCTGGTGGATGTGCTTGATGG), light-blue highlighted: PAM-sequence, light-green highlighted: homozygous GT deletion, underlined, bold-red letters: homozygous nucleotide insertions, hom = homozygous, het = heterozygous, $?=$ not definable.

\begin{tabular}{|c|c|c|}
\hline Lines & Potri.014G102600 (“TAC-14”): Genomic Target Region 2R & Type of Mutation \\
\hline $\begin{array}{l}\text { P1, not-transgenic } \\
\text { regenerated lines * }\end{array}$ & ......GAAACAAGTAGC CCT GGT-GGATGTGCTTGATGGTTG . . . . . & Wildtype \\
\hline N499-1-2 & ...... GAAACAAGTAGCCCTG - - - GGATGTGCTTGATGGTTG . . . . . & GT-deletion, hom \\
\hline $\begin{array}{l}\text { N499-2-1, }-3-1,-10-2 \\
\quad-14-2,-17-1\end{array}$ & ... ... GAAACAAGTAGCCCTGGTTGGATGTGCTTGATGGTTG . . . . . . & T-insertion, hom \\
\hline N499-3-3 & ...... GAAACAAGTAGCCCTGGTGGGATGTGCTTGATGGTTG . . . . . . & G-insertion, hom \\
\hline N499-10-3 & ...... GAAACAAGTAGCCCTGGTAGGATGTGCTTGATGGTTG . . . . . . & A-insertion, hom \\
\hline N499-4-1, -8-3, -11-1 & ...... GAAACAAGTAGCCCTGGTKGGATGTGCTTGATGGTTG . & G/T-insertion, hom \\
\hline N499-13-2 & $\begin{array}{c}\text {... . . GAAACAAGTAGCCCTGGT-GGATGTGCTTGATGGTTG . . . . . . } \\
\qquad . \ldots \text { ????????????????? . . . . }\end{array}$ & $\begin{array}{l}\text { ? bp insertion or } \\
\text { deletion, het }\end{array}$ \\
\hline
\end{tabular}

Potri.002G175300 (“TAC-2”): Genomic Target Region 2R

\begin{tabular}{|c|c|c|}
\hline $\begin{array}{l}\mathrm{P} 1 \text {, not-transgenic } \\
\text { regenerated lines * }\end{array}$ & ... ... GAAACAAGTAGC CCT GGT-GGATGTGCTTGATGGTTG . . . . . & Wildtype \\
\hline N499-1-2 & . . . . . GAAACAAGTAGCCCTGGTGGGATGTGCTTGATGGTTG . . . . . . & G-insertion, hom \\
\hline N499-10-2 & ... . . . GAAACAAGTAGCCCTGGTAGGATGTGCTTGATGGTTG . . . . . . . & A-insertion, hom \\
\hline N499-2-1, -3-1, -8-3, -10-3 & ... ... GAAACAAGTAGCCCTGGTRGGATGTGCTTGATGGTTG . . . . . . & A/G-insertion, hom \\
\hline $\begin{array}{l}\text { N499-4-1, -11-1, -13-2, } \\
\quad-14-2,-17-1\end{array}$ & $\begin{array}{c}\ldots \ldots \text { GAAACAAGTAGCCCTGGT-GGATGTGCTTGATGGTTG } \ldots \ldots \ldots \\
\ldots \ldots \text { ? } \ldots \text { ????????????????? . . . . . }\end{array}$ & $\begin{array}{l}\text { ? bp insertion or } \\
\text { deletion, het }\end{array}$ \\
\hline
\end{tabular}

With exception of lines N499-5, -7, 12, and -15, the N499-lines were also analyzed for putative CRISPR/Cas9-caused mutations in the targets 2R and 4F of Potri.002G175300. Again, not a single mutation could be found for target $4 \mathrm{~F}$, but all transgenic lines carry mutations in target $2 \mathrm{R}$ (Tables 2 and 3). The preferential homozygous modifications in target 2R of Potri.002G175300 were a single insertion of either A or G (lines N499-1-2, -10-2; Tables 2 and 3) or of different nucleotides (mostly $\mathrm{R}$ and once $\mathrm{K}$ ) in both allelic strands (lines N499-2-1, -3-1, and -8-3; Tables 2 and 3). In lines N499-4-1, -11-1, -13-2, -14-2, -17-1, heterozygous insertions or deletions were detected, which were unfortunately undefinable in most of these lines (Tables 2 and 3).

Taken together, the editing results in the target regions of the two $P$. trichocarpa putative PpeTAC1 paralogs in $P . \times$ canescens, lines N499-1-2, -2-1, -3-1, -8-3, -10-2, and -19-1 reveal homozygous single or double nucleotide insertions/deletions putatively leading to knockout mutations in both genes (bold in Potri in Table 2). On the other hand, lines N499-4-1, $-11-1,-14-2$, and -17-1 show homozygous mutations in Potri.014G102600 but heterozygous mutations in Potri.002G175300 (grey shaded, bold, and underlined in Potri in Table 2). And finally, line N499-13-2 reveals heterozygous mutations in both genes (underlined in Potri in Table 2).

\subsection{Morphological Investigations of In Vitro and Glasshouse Grown Plants}

During the growth of obtained putative transgenic N499-lines in vitro in cultivation boxes, a plant habitus with upright oriented leaves, indicating a smaller petiole angle, appeared in some lines (Figure 1a,b). However, this possible change of the plant phenotypes in vitro could not be quantified. Therefore, lines N499-1-2, -2-1,-3-1, -8-3, and -10-2 with 
CRISPR/Cas9-mediated homozygous mutations in both P. trichocarpa paralogous genes Potri.014G102600 and Potri.002G175300 were transferred to soil and cultivated in the glasshouse for morphological inspections. In addition, lines N499-4-1, -11-1, -14-2, and -17-1 with a homozygous knock-out mutation in just Potri.014G102600 but a heterozygous one in Potri.002G175300 and line N499-13-2 with heterozygous mutations in both genes were also transferred to the glasshouse. Finally, the wildtype P1 and two regenerated but not-transgenic lines (without TAC1-mutation; N499-6-1, and -9-1) were included as controls in the experimentally tests in the glasshouse (lines transferred to the glasshouse are grey-shaded in the first column of Table 2).

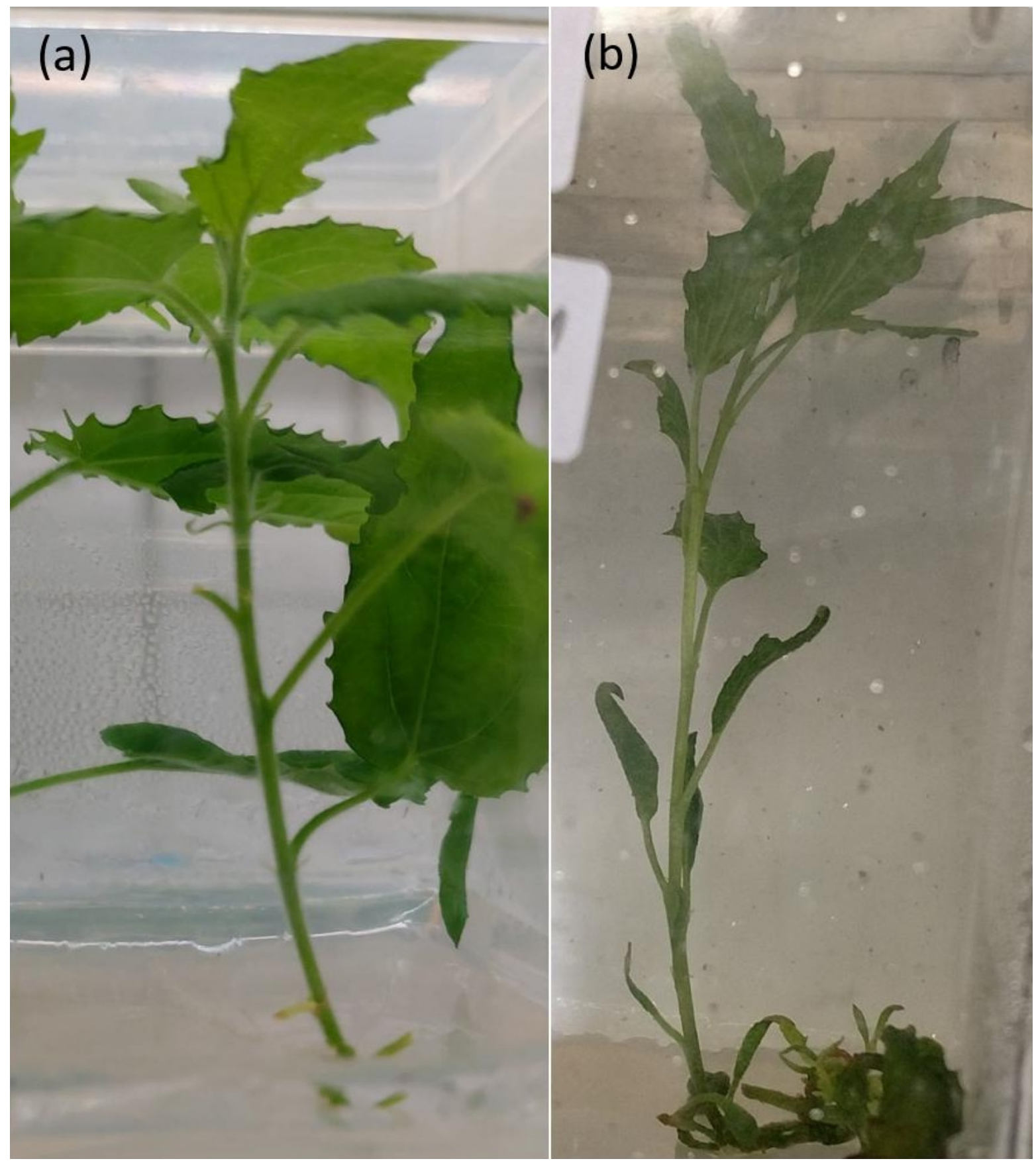

Figure 1. Representative poplar plants grown in vitro. (a) Not-transgenic control plant of $P . \times$ canescens clone INRA 717-1B4. (b) Plant with upright oriented leaves carrying a homozygous knock-out editing in both of the poplar Potri.014G102600 ("TAC-14"; orthologous to the rice TILLER ANGLE CONTROL 1, (TAC1) and its paralog Potri.002G175300 ("TAC-2"). 
In the first year (2019), because of the lack of side-shoots, angles of representative leaf petioles were recorded from five plants of each of P1 wildtype and the two regenerated but not-transgenic controls, and the transgenic edited lines after two months' growth in the glasshouse (July 2019) (Figures 2a,b and 3a). Significantly smaller petiole angles (indicating more up-right growth) were found in all lines with homozygous knock-out in the two P. trichocarpa putative PpeTAC1 paralogs in P. $\times$ canescens (N499-1-2, -2-1, -3-1, $-4-1,-8-3$, and -10-2) compared to the control clones (P1 and the regenerated but nottransgenic lines N499-6-1, and -9-1) (Figure 3a). In lines that were only homozygous in Potri.014G102600 but heterozygous in Potri.002G175300, the situation turned out to be inconsistent. Lines N499-4-1 and -17-1 reveal significantly smaller petiole angles, however, in lines N499-11-1 and -14-2, petiole angles were also reduced but not significant. In the line that is heterozygous in both genes (N499-13-2), petiole angles turned out to be slightly reduced or similar to the controls (Figure 3a).

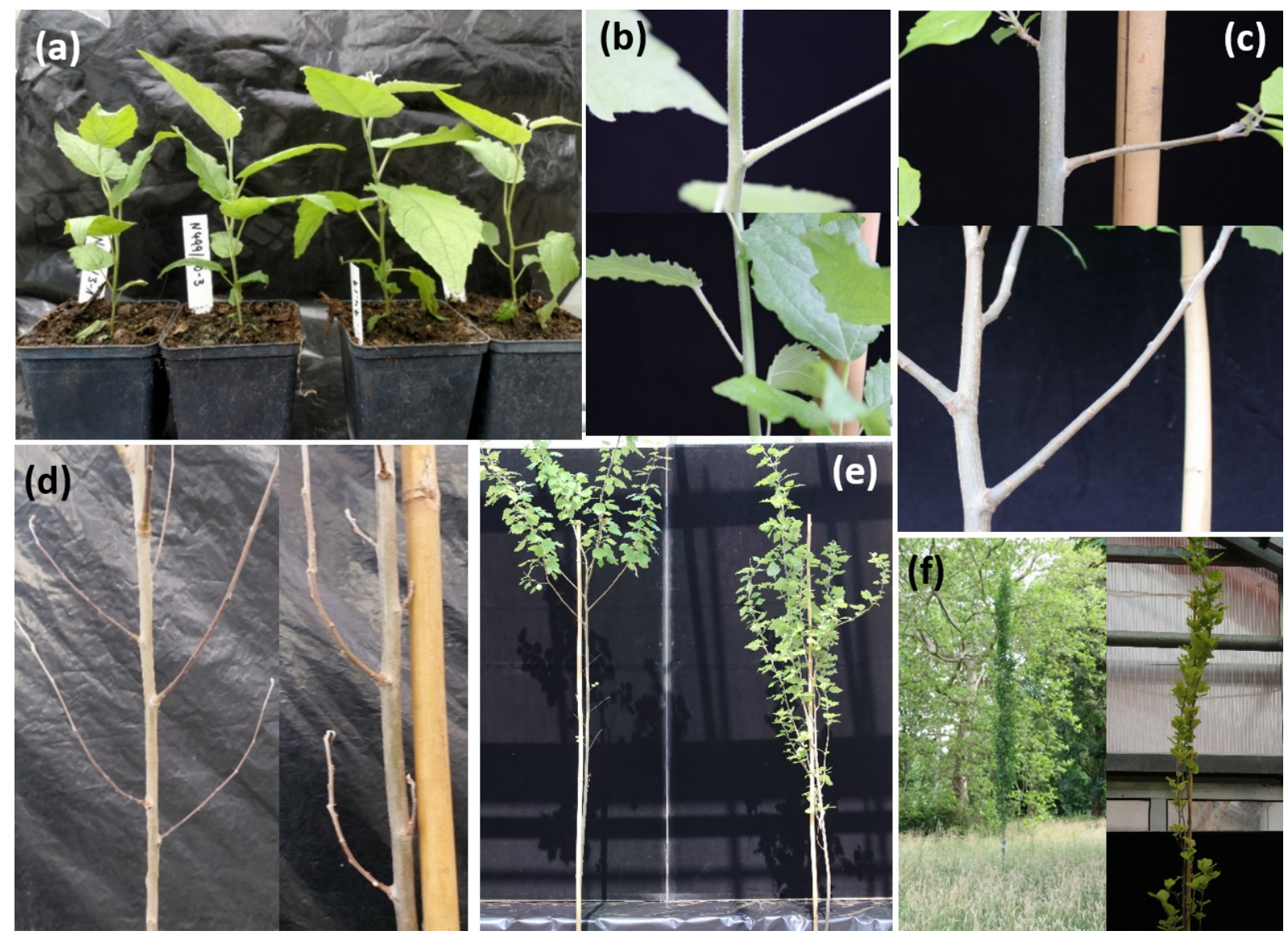

Figure 2. Representative poplar plants grown in the glasshouse and in the field. (a) Transgenic plants of N499-3-1 and N499-10-3 (left two plants) with slightly upright oriented leaves in comparison with two INRA 717-1B4 control plants (right) after 1 month of cultivation in soil (2019). (b) Representative petioles of a control (upper image) and a homozygous "TAC-14" / "TAC-2" knock-out edited N499-transgenic plant (lower image) during growth in the first year in the glasshouse (2019). (c) Representative side-shoots of a control (upper image) and a homozygous TAC-14/TAC-2 knock-out edited N499-transgenic plant (lower image) during growth in the second year in the glasshouse (2020). (d) Control (left) and homozygous "TAC-14" /"TAC-2" knock-out edited N499-transgenic plant in a glasshouse during the winter season after the second year (2020/21) of growth in a glasshouse. (e) Representative control (left) and homozygous "TAC-14" /"TAC-2" knock-out edited N499-transgenic plant during growth in the second year in the glasshouse (2020). (f) Erect $P$. tremula mutant grown in the field (left) compared to a homozygous "TAC-14" /"TAC-2" knock-out edited N499-transgenic plant during growth in the third year in the glasshouse (2021). Note: N499-transgenic plants with a homozygous editing event only in "TAC-14" revealed also a pronounced upright growth of side-shoots. 

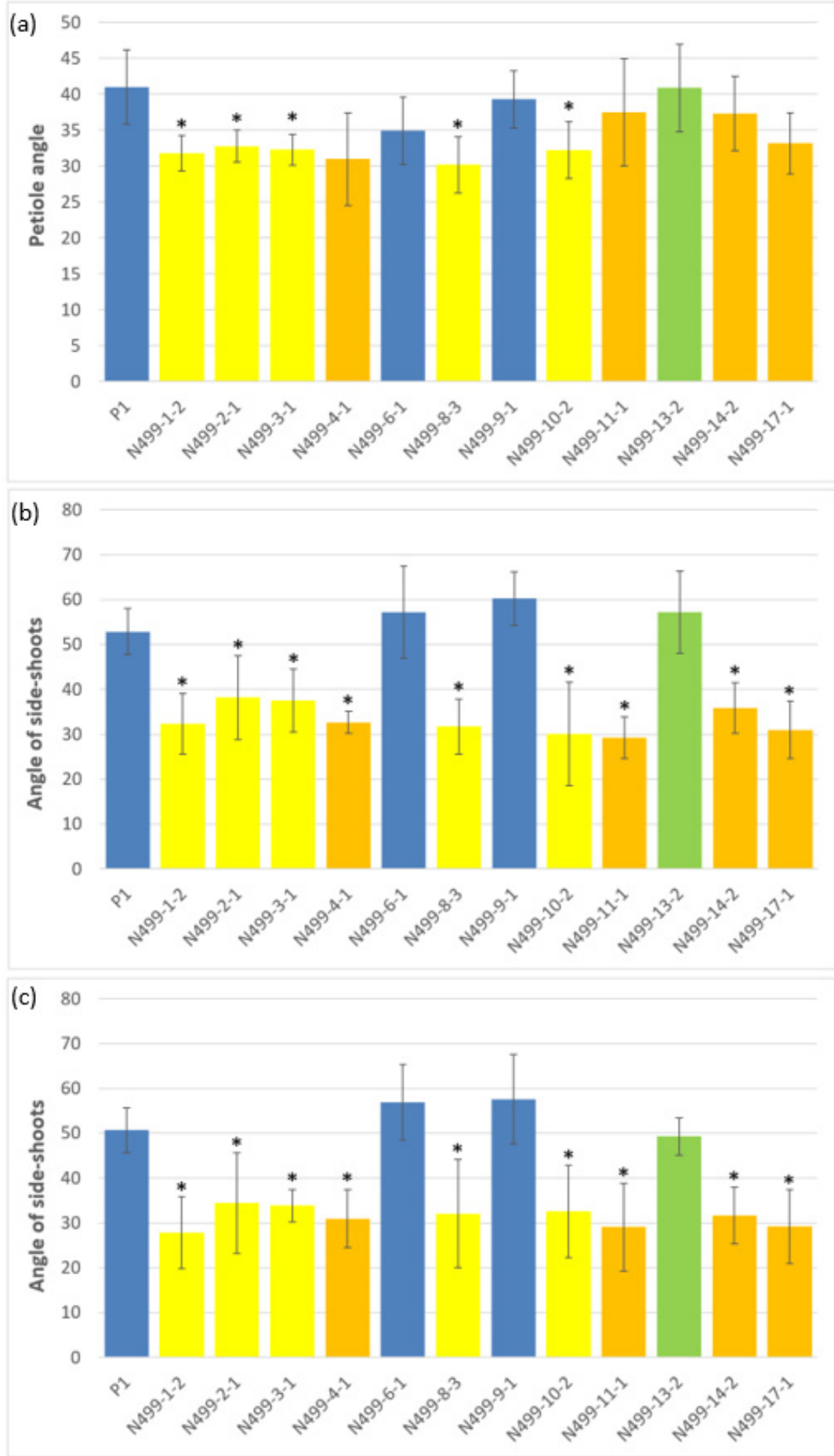

Figure 3. Measurements of the leaf petiole angles in the first year (2019 (a), and angles of side-shoots of plants of different independent N499 edited-transgenic lines and not-transgenic control plants grown in a glasshouse in the second (2020) (b) and third year (2021) (c) after transfer, respectively. Edited transgenic N499-lines reveal either homo- knock-out editing of both Potri.014G102600 ("TAC14") and its paralog Potri.002G175300 ("TAC-2") (yellow-columns), homozygous in "TAC-14" but heterozygous in "TAC-2" (orange columns) or heterozygous in both TAC-genes (green columns). Controls: P1 and not-transgenic lines (blue columns). Angles of leaf petioles and side-shoots were determined from five plants of each line. Error bars represent the standard deviations of three biological replicates derived from five independent trees. ${ }^{*}=$ Values differ significantly at $p<0.05$ (Fisher's F-test) from the control P1. 
Angles of representative side-shoots were determined in the second and third year (August 2020, end of April 2021) of glasshouse grown plants, again from five plants of the transgenic and of different control lines (Figure 2c,d and Figure $3 b, c)$. Here, results again show that all lines with homozygous knock-out in the two P. trichocarpa putative PpeTAC1 paralogs in $P$. $\times$ canescens but also all lines that are homozygous just in Potri.014G102600 but heterozygous in Potri.002G175300 show significantly smaller angles of the side-shoots than the P1 and the N499 control clones. Only the line N499-13-2, heterozygous in both genes, revealed side-shoot angles similar to the control lines. The phenotype of the homozygous Potri.014G102600 knock-out plants revealed an erect growth compared to the control plants (Figure 2e), and in particular, in the third year of glasshouse growth, the Potri.014G102600 knock-out plants are phenotypically quite similar to unrelated erect $P$. tremula individuals grown in the field (Figure $2 \mathrm{f}$ ).

Total height and diameter of the main shoot $(1 \mathrm{~cm}$ above soil $)$ measured from all P1, N499-control and N499-transgenic plants after terminal bud formation in the first (January 2020) and the second year (February 2021) of cultivation in the glasshouse revealed no significant differences (data not shown).

\section{Discussion and Conclusions}

Upright or pyramidal growth of plants is a pronounced phenotype found in many plant species, e.g., Prunus persica (peach) [40], Cupressus sempervirens 'Pyramidalis' (Italian Cypress [45], Taxus baccata 'Pyramidalis' (pillar yew [46]), Populus tremula 'Erecta' (Swedish columnar aspen [47]), P. alba var. pyramidalis (P. bolleana [48]), and P. nigra 'Italica' (Lombardy poplar [49]), but also, a commercial-used hybrid poplar cultivar named Sundancer ${ }^{\mathrm{TM}}$ reveals vertical growth of branches [50]. Also, quite recently Fladung (unpublished) noticed a pyramidal growth in an aspen, $P$. tremula, male individual growing in the arboretum of the Thuenen Institute of Forest Genetics, Grosshansdorf, Germany. Control of branching, axillary meristem initiation, and the outgrowth of axillary buds have been intensively studied at the molecular level [62]. However, the molecular basis for different tree-growth habits and mechanisms determining the branch angle is still poorly understood, despite its possible importance for practical applications.

Among the poplar genotypes, without doubt, the most famous upright-growing tree mutant is P. nigra 'Italica' (Lombardy poplar), a cultivated clonal variety distributed worldwide for more than 300 years $[49,52,63]$. Even if the origin of the mutation is uncertain, the mutation was discovered in a single male P. nigra tree grown in central Asia [49]. In peach, a mutation in the orthologous gene of the rice TILLER ANGLE CONTROL 1 (TAC1) was shown to be responsible for pyramidal plant growth [43]. However, the occurrence of the TAC1 was, besides some other genes, originally thought to be restricted to monocots [64,65]. But TAC1 homologs were soon identified as well in dicots [18,43], and overexpression of the PdTAC1 gene in peach has led to more horizontal oriented branches [44]. Also [43] proposed that the pyramidal phenotype of the P. nigra, Lombardy poplar, is possibly caused by a mutation in a poplar ortholog of the TAC1 gene. However, so far, not for any pyramidal tree genotype, has it experimentally been confirmed that a knock-out mutation of the rice TAC1 orthologous gene(s) leads to pyramidal plant growth

To experimentally test the role of putative $T A C 1$ ortholog(s) in poplar, we first determined in silico the most homologous gene and its paralogous one in P. trichocarpa, namely Potri.014G102600 ("TAC-14") and Potri.002G175300 ("TAC-2"), respectively. The orthologous genes were also determined in both $P$. tremula and $P$. $\times$ canescens by employing the two obtained P. trichocarpa sequences. Based on all sequences obtained from the three poplar species, two guide RNAs (gRNAs) were designed to generate TAC1 mutations by use of the CRISPR/Cas9 system. We obtained a number of CRISPR/Cas9-mediated homozygous and heterozygous mutations in the $P . \times$ canescens clone INRA 717-1B4 in one or both of the TAC1 genes. The TAC1-edited lines and (transgenic and not-transgenic) controls were analyzed molecularly and inspected morphologically for three years after transfer to the glasshouse. As an indicator for pyramidal growth, we focused on the prominent features, 
leaf and branch angle, which makes a major contribution to the overall tree architecture [66]. Our results indicate that the homozygous knock-out of just "TAC-14" is sufficient to induce pyramidal plant growth in $P$. $\times$ canescens.

During in vitro culture, a possible change of the plant phenotype towards pyramidal growth could be noticed but not quantified. Following transfer of the lines into the glasshouse, lines with CRISPR/Cas9-mediated homozygous mutations in both "TAC-14" and "TAC-2" genes formed leaves with a narrower angle and showed an upright growth of shoots in the second year of glasshouse cultivation. This finding is in agreement with the results in peach, where mutant varieties with pronounced vertical growth of branches or upright stems have been associated with a loss-of-function mutation in the PdTAC1 gene [43]. In peach, but also in other woody plant species, obvious benefits of upright growth could be e.g., reduced need for pruning, easier application of chemicals (if needed), automation of harvest, and increased yield simply through high density planting [40,41]. Further [38] could show that erect-leaf habit allows more efficient penetration of light into lower canopy layers, thus increasing total plant net photosynthesis potentially leading to increased biomass formation. However, our data on plant height and stem diameter of poplar grown for three years in the glasshouse (of course not comparable to field growth conditions) revealed no significant difference between pyramidal and control plants.

Even though no increased biomass (just taking the two growth parameters height and stem diameter into account) could be noticed for pyramidal poplar, these individuals are, at least, similarly "productive" as "normal"-growing ones. This fact could be of high interest for short rotation coppices (SRCs), because due to erect leaf or shoot growth, pyramidal plants need reduced individual space, and thus, a higher number of plants could be planted on a default field size of SRCs, as e.g., reported for peach [40] and apple [41]. This could lead in consequence to increased yield per ha land area without any breeding activities. However, interestingly, with the possible exception of the hybrid poplar cultivar Sundancer ${ }^{\mathrm{TM}}$ [50], so far, all commercially used poplar clones revealed a non-pyramidal plant growth. However, theoretically at least, any commercially used poplar clone could serve as starting material for CRISPR-based "TAC-14"-knock-out to produce near-isogenic lines with pyramidal growth but unchanged yield. Unfortunately, in July 2018, the European Court of Justice (Case C-528/16) has judged that all plants derived by CRISPR-based modifications are to be regarded as genetically modified organisms (GMOs) within the meaning of Directive 2001/18 [67]. However, as a first step, already available erect poplar genotypes can be tested for growth and yield parameters because SRCs are replicable everywhere that the site conditions support poplar trees.

Supplementary Materials: The following are available online at https:/ / www.mdpi.com/article/ 10.3390/f12121615/s1, Figure S1: C672p9ioR-35sCasWT-Nuc plasmid, Table S1: Putative PpeTAC1 (EMBL acc.no. KF218366; [43]) homologous genes in P. trichocarpa., Table S2: Primers used in this study.

Funding: The project was funded with the internal budget of the Thünen Institute of Forest Genetics.

Acknowledgments: I deeply thank Katrin Groppe for tremendous technical assistance in the laboratory and the glasshouse staff (Wolfgang Graf, Sabine Benischek, Monika Spauszus) for plant cultivation and taking measurements.

Conflicts of Interest: The author of the research article has no conflict of interest to disclose.

\section{References}

1. Reinhardt, D.; Kuhlemeier, C. Plant architecture. EMBO Rep. 2002, 3, 846-851. [CrossRef]

2. Fladung, M.; Bossinger, G.; Roeb, G.W.; Salamini, F. Correlated alterations in leaf and flower morphology and rate of photosynthesis in a midribless (mbl) mutant of Panicum maximum (Jacq.). Planta 1991, 184, 356-361. [CrossRef]

3. Fladung, M. Genetic variants of Panicum maximum (Jacq.) in C4 photosynthetic traits. J. Plant Physiol. 1994, 143, 165-172. [CrossRef]

4. Godin, C. Representing and encoding plant architecture: A review. Ann. For. Sci. 2000, 57, 413-438. [CrossRef]

5. Wang, Y.; Li, J. Molecular basis of plant architecture. Ann. Rev. Plant Biol. 2008, 59, 253-279. [CrossRef] 
6. Costes, E. Physiology and genetics of plant architecture. Ann. Plant Rev. Online 2019, 2, 1031-1068.

7. Barthélémy, D.; Caraglio, Y. Plant architecture: A dynamic, multilevel and comprehensive approach to plant form, structure and ontogeny. Ann. Bot. 2007, 99, 375-407. [CrossRef]

8. Chelakkot, R.; Mahadevan, L. On the growth and form of shoots. J. R. Soc. Interface 2017, 14, 20170001. [CrossRef]

9. Nybom, N. Mutation types in barley. Acta Agricult. Scand. 1954, 4, 430-456. [CrossRef]

10. Kharkwal, M.C.; Pandey, R.N.; Pawar, S.E. Mutation breeding for crop improvement. In Plant Breeding; Jain, H.K., Kharkwal, M.C., Eds.; Springer: Dordrecht, The Netherlands, 2004; pp. 601-645.

11. Pathirana, R. Plant mutation breeding in agriculture. Plant Sci. Rev. 2011, 6, 107-126. [CrossRef]

12. Oladosu, Y.; Rafii, M.Y.; Abdullah, N.; Hussin, G.; Ramli, A.; Rahim, H.A.; Miah, G.; Usman, M. Principle and application of plant mutagenesis in crop improvement: A review. Biotechnol. Biotechnolog. Equip. 2016, 30, 1-16. [CrossRef]

13. Holme, I.B.; Gregersen, P.L.; Brinch-Pedersen, H. Induced genetic variation in crop plants by random or targeted mutagenesis: Convergence and differences. Front. Plant Sci. 2019, 10, 1468. [CrossRef]

14. Lamo, K.; Bhat, D.J.; Kour, K.; Solanki, S.P.S. Mutation studies in fruit crops: A review. Int. J. Curr. Microbiol. Appl. Sci. 2017, 6, 3620-3633. [CrossRef]

15. Fladung, M.; Ziegenhagen, B. M13 DNA fingerprinting can be used in studies on phenotypical revisions of forest tree mutants. Trees 1998, 12, 310-314. [CrossRef]

16. Fladung, M.; Tusch, A.; Markussen, T.; Ziegenhagen, B. Analysis of morphological mutants in Picea. In Proceedings of the Applications of Biotechnology to Forest Genetics (Biofor 99), Vitoria-Gasteiz, Spain, 22-25 September 1999; Espinel, S., Ritter, E., Eds.; pp. 167-170.

17. Flachowsky, H.; Hanke, M.V.; Peil, A.; Strauss, S.H.; Fladung, M. A review on transgenic approaches to accelerate breeding of woody plants. Plant Breed. 2009, 128, 217-226. [CrossRef]

18. Teichmann, T.; Muhr, M. Shaping plant architecture. Front. Plant Sci. 2015, 6, 233. [CrossRef]

19. Zawaski, C.; Kadmiel, M.; Pickens, J.; Ma, C.; Strauss, S.H.; Busov, V. Repression of gibberellin biosynthesis or signaling produces striking alterations in poplar growth, morphology, and flowering. Planta 2011, 234, 1285-1298. [CrossRef]

20. Buhl, C.; Strauss, S.H.; Lindroth, R.L. Genetic down-regulation of gibberellin results in semi-dwarf poplar but few non-target effects on chemical resistance and tolerance to defoliation. J. Plant Ecol. 2019, 12, 124-136. [CrossRef]

21. Busov, V.B.; Meilan, R.; Pearce, D.W.; Ma, C.; Rood, S.B.; Strauss, S.H. Activation tagging of a dominant gibberellin catabolism gene (GA 2-oxidase) from poplar that regulates tree stature. Plant Physiol. 2003, 132, 1283-1291. [CrossRef]

22. Weigel, D.; Nilsson, O. A developmental switch sufficient for flower initiation in diverse plants. Nature 1995, 377, 495-500. [CrossRef]

23. Rottmann, W.H.; Meilan, R.; Sheppard, L.A.; Brunner, A.M.; Skinner, J.S.; Ma, C.; Cheng, S.; Jouanin, L.; Pilate, G.; Strauss, S.H. Diverse effects of overexpression of LEAFY and PTLF, a poplar (Populus) homolog of LEAFY/FLORICAULA, in transgenic poplar and Arabidopsis. Plant J. 2000, 22, 235-245. [CrossRef]

24. Yuan, H.M.; Chen, S.; Lin, L.; Wei, R.; Li, H.Y.; Liu, G.F.; Jiang, J. Genome-wide analysis of a TaLEA-introduced transgenic Populus simonii $\times$ Populus nigra dwarf mutant. Int. J. Mol. Sci. 2012, 13, 2744-2762. [CrossRef]

25. Bruegmann, T.; Fladung, M. Overexpression of both flowering time genes AtSOC1 and SaFUL revealed huge influence onto plant habitus in poplar. Tree Genet. Genom. 2019, 15, 1-13. [CrossRef]

26. Fladung, M. Transformation of diploid and tetraploid potato clones with the rolC gene of Agrobacterium rhizogenes and characterization of transgenic plants. Plant Breed. 1990, 104, 295-304. [CrossRef]

27. Schmülling, T.; Fladung, M.; Großmann, K.; Schell, J. Hormonal content and sensitivity of transgenic tobacco and potato plants expressing single rol genes of Agrobacterium rhizogenes T-DNA. Plant J. 1993, 3, 371-382. [CrossRef]

28. Fladung, M.; Muhs, H.J.; Ahuja, M.R. Morphological changes observed in transgenic Populus carrying the rolC gene from Agrobacterium rhizogenes. Silv. Genet. 1996, 45, 349-354.

29. Fladung, M.; Kumar, S.; Ahuja, M.R. Genetic transformation of Populus genotypes with different chimaeric gene constructs: Transformation efficiency and molecular analysis. Transgen. Res. 1997, 6, 111-121. [CrossRef]

30. Fladung, M.; Polak, O. Ac/Ds-transposon activation tagging in poplar: A powerful tool for gene discovery. BMC Genom. 2012, 13, 61. [CrossRef] [PubMed]

31. Eriksson, M.E.; Israelsson, M.; Olsson, O.; Moritz, T. Increased gibberellin biosynthesis in transgenic trees promotes growth, biomass production and xylem fiber length. Nat. Biotechol. 2000, 18, 784-788. [CrossRef]

32. Dünisch, O.; Funada, R.; Nakaba, S.; Fladung, M. Influence of overexpression of a gibberellin 20-oxidase gene on the kinetics of xylem cell development in hybrid poplar (Populus tremula $L \times$ P. tremuloides Michx). Holzforschung 2006, 60, 608-617. [CrossRef]

33. Jeon, H.W.; Cho, J.S.; Park, E.J.; Han, K.H.; Choi, Y.I.; Ko, J.H. Developing xylem-preferential expression of PdGA20ox1, a gibberellin 20-oxidase 1 from Pinus densiflora, improves woody biomass production in a hybrid poplar. Plant Biotechnol. J. 2016, 14, 1161-1170. [CrossRef] [PubMed]

34. Bruegmann, T.; Wetzel, H.; Hettrich, K.; Smeds, A.; Willför, S.; Kersten, B.; Fladung, M. Knockdown of PCBER1, a gene of neolignan biosynthesis, resulted in increased poplar growth. Planta 2019, 249, 515-525. [CrossRef]

35. Sakamoto, T.; Morinaka, Y.; Ohnishi, T.; Sunohara, H.; Fujioka, S.; Ueguchi-Tanaka, M.; Mizutani, M.; Sakata, K.; Takatsuto, S.; Yoshida, S.; et al. Erect leaves caused by brassinosteroid deficiency increase biomass production and grain yield in rice. Nat. Biotechnol. 2006, 24, 105-109. [CrossRef] 
36. Richards, R.A.; Cavanagh, C.R.; Riffkin, P. Selection for erect canopy architecture can increase yield and biomass of spring wheat. Field Crops Res. 2019, 244, 107649. [CrossRef]

37. Burgess, A.J.; Retkute, R.; Herman, T.; Murchie, E.H. Exploring relationships between canopy architecture, light distribution, and photosynthesis in contrasting rice genotypes using 3D canopy reconstruction. Front. Plant Sci. 2017, 8, 734. [CrossRef]

38. Mantilla-Perez, M.B.; Salas Fernandez, M.G. Differential manipulation of leaf angle throughout the canopy: Current status and prospects. J. Exp. Bot. 2017, 68, 5699-5717. [CrossRef] [PubMed]

39. Sinclair, T.R.; Sheehy, J.E. Erect leaves and photosynthesis in rice. Science 1999, 283, 1456. [CrossRef]

40. Scorza, R.; Lightner, G.W.; Liverani, A. The pillar peach tree and growth habit analysis of compact x pillar progeny. J. Amer. Soc. Hortic. Sci. 1989, 114, 991-995.

41. Kelsey, D.F.; Brown, S.K. 'McIntosh Wijcik': A columnar mutation of 'McIntosh' apple proving useful in physiology and breeding research. Fruit Var. J. 1992, 46, 83-87.

42. Tworkoski, T.; Scorza, R. Root and shoot characteristics of peach trees with different growth habits. J. Amer. Soc. Hortic. Sci. 2001, 126, 785-790. [CrossRef]

43. Dardick, C.; Callahan, A.; Horn, R.; Ruiz, K.B.; Zhebentyayeva, T.; Hollender, C.; Whitaker, M.; Abbott, A.; Scorza, R. PpeTAC1 promotes the horizontal growth of branches in peach trees and is a member of a functionally conserved gene family found in diverse plants species. Plant J. 2013, 75, 618-630. [CrossRef] [PubMed]

44. Hollender, C.A.; Waite, J.M.; Tabb, A.; Raines, D.; Chinnithambi, S.; Dardick, C. Alteration of TAC1 expression in Prunus species leads to pleiotropic shoot phenotypes. Horticult. Res. 2018, 5, 1-9. [CrossRef]

45. Papageorgiou, A.C.; Panetsos, K.P.; Hattemer, H.H. Genetic differentiation of natural Mediterranean cypress (Cupressus sempervirens L.) populations in Greece. For. Genet. 1994, 1, 1-12.

46. Hoffman, M.H.A. Cultivar classification of Taxus L. (Taxaceae). Acta Horticult. 2004, 634, 91-96. [CrossRef]

47. Kwantlen Polytechnic University. School of Horticulture Plant Database-Populus tremula 'Erecta'. Available online: https: / / plantdatabase.kpu.ca/plant/plantDetail/284 (accessed on 22 November 2021).

48. Xu, X.; Tong, L.; Li, F.; Kang, S.; Qu, Y. Sap flow of irrigated Populus alba var. pyramidalis and its relationship with environmental factors and leaf area index in an arid region of Northwest China. J. For. Res. 2011, 16, 144-152. [CrossRef]

49. Zsuffa, L. The genetics of Populus nigra L. Ann. For. 1974, 6, 29-53.

50. Schroeder, W.; Soolanayakanahally, R.; Lindquist, C. AC Sundancer ${ }^{\mathrm{TM}}$ Poplar. Can. J. Plant Sci. 2013, 93, 1285-1287. [CrossRef]

51. Wood, C.D. "A Most Dangerous Tree": The Lombardy Poplar in landscape gardening. Arnoldia 1994, 54, 24-30.

52. Vanden Broeck, A.; Cox, K.; Brys, R.; Castiglione, S.; Cicatelli, A.; Guarino, F.; Heinze, B.; Steenackers, M.; Vander Mijnsbrugge, K. Variability in DNA methylation and generational plasticity in the Lombardy Poplar, a single genotype worldwide distributed since the Eighteenth century. Front. Plant Sci. 2018, 9, 1635. [CrossRef]

53. Tuskan, G.A.; DiFazio, S.; Jansson, S.; Bohlmann, J.; Grigoriev, I.; Hellsten, U.; Putnam, N.; Ralph, S.; Rombauts, S.; Salamov, A.; et al. The genome of black cottonwood, Populus trichocarpa (Torr. \& Gray). Science 2006, 313, 1596-1604.

54. Bruegmann, T.; Deecke, K.; Fladung, M. Evaluating the efficiency of gRNAs in CRISPR/Cas9 mediated genome editing in poplars. Int. J. Mol. Sci. 2019, 20, 3623. [CrossRef]

55. Leple, J.C.; Brasileiro, A.C.M.; Michel, M.F.; Delmotte, F.; Jouanin, L. Transgenic poplars: Expression of chimeric genes using four different constructs. Plant Cell Rep. 1992, 11, 137-141. [CrossRef]

56. Briones, M.V.; Hoenicka, H.; Cañas, L.A.; Beltrán, J.P.; Hanelt, D.; Sharry, S.; Fladung, M. Efficient evaluation of a gene containment system for poplar through early flowering induction. Plant Cell Rep. 2020, 39, 577-587. [CrossRef] [PubMed]

57. Hofacker, I.L. Vienna RNA secondary structure server. Nucl. Acids Res. 2003, 31, 3429-3431. [CrossRef] [PubMed]

58. Andronescu, M.; Condon, A.; Hoos, H.H.; Mathews, D.H.; Murphy, K.P. Efficient parameter estimation for RNA secondary structure prediction. Bioinformatics 2007, 23, i19-i28. [CrossRef] [PubMed]

59. Zuker, M.; Stiegler, P. Optimal computer folding of large RNA sequences using thermodynamics and auxiliary information. Nucl. Acids Res. 1981, 9, 133-148. [CrossRef]

60. Mader, M.; Le Paslier, M.C.; Bounon, R.; Bérard, A.; Faivre Rampant, P.; Fladung, M.; Leplé, J.C.; Kersten, B. Whole-genome draft assembly of Populus tremula $\times$ Populus alba clone INRA 717-1B4. Silv. Genet. 2016, 65, 74-79. [CrossRef]

61. Bruegmann, T.; Polak, O.; Deecke, K.; Nietsch, J.; Fladung, M. Poplar Transformation. In Transgenic Plants; Kumar, S., Barone, P., Smith, M., Eds.; Methods in Molecular Biology; Humana Press: New York, NY, USA, 2019; pp. 165-177.

62. Bennett, T.; Leyser, O. Something on the side: Axillary meristems and plant development. Plant Mol. Biol. 2006, 60, 843-854. [CrossRef]

63. Henry, A. The Black poplars. Gard. Chron. 1914, 56, 46-47.

64. Doebley, J.; Wang, R.L. Genetics and the evolution of plant form: An example from maize. Cold Spring Harbor Symp. Quant. Biol. 1997, 62, 361-367.

65. Yu, B.; Lin, Z.; Li, H.; Li, X.; Li, J.; Wang, Y.; Zhang, X.; Zhu, Z.; Zhai, W.; Wang, X.; et al. TAC1, a major quantitative trait locus controlling tiller angle in rice. Plant J. 2007, 52, 891-898. [CrossRef] [PubMed]

66. Hallé, F.; Oldeman, R.A.; Tomlinson, P.B. Opportunistic tree architecture. In Tropical Trees and Forests; Hallé, F., Oldeman, R.A., Tomlinson, P.B., Eds.; Springer: Berlin/Heidelberg, Germany, 1978; pp. 269-331.

67. Carreño, I.; Dolle, T. The Court of Justice of the European Union's judgment on mutagenesis and international trade: A case of GMO, mutagenesis and international trade. Glob. Trade Cust. J. 2019, 14, 91-101. 